\title{
Téoros
}

Revue de recherche en tourisme

\section{France - Amérique du Nord : des flux... fluctuants}

\section{Pierre Bellerose}

Volume 8, numéro 1, mars 1989

France-Québec

URI : https://id.erudit.org/iderudit/1080348ar

DOI : https://doi.org/10.7202/1080348ar

Aller au sommaire du numéro

Éditeur(s)

Université du Québec à Montréal

ISSN

0712-8657 (imprimé)

1923-2705 (numérique)

Découvrir la revue

Citer cet article

Bellerose, P. (1989). France - Amérique du Nord : des flux... fluctuants. Téoros, 8(1), 6-7. https://doi.org/10.7202/1080348ar d'utilisation que vous pouvez consulter en ligne.

https://apropos.erudit.org/fr/usagers/politique-dutilisation/ 


\section{France - Amérique du Nord: des flux... fluctuants}

La situation du tourisme en France et en Amérique du Nord (États-Unis, Canada et Québec) est très différente. En effet, malgré le fait qu'on pourrait placer trois fois la France dans le Québec, la France est le plus grand pays de l'Europe de l'Ouest. Son territoire de $547000 \mathrm{~km}^{2}$ excède ceux du Royaume-Uni, de l'Allemagne de l'Ouest et de la Hollande combines. Pourtant la population française ne représente même pas la moitié de la population de ces trois pays réunis. On retrouve donc très près de la France un marché potentiel immense. Une autre clef de cette différence est Paris. En effet, cette capitale est facilement accessible pour plusieurs pays émetteurs de touristes de l'Europe de l'Ouest; Paris se trouve approximativement à mi-chemin entre Édimbourg et Rome tout en étant relié à tous les pays européens par un vaste et efficace réseau de service routier, ferroviaire et aérien. Paris est aussi le centre numéro un mondial des conférences et congrès.

A l'inverse, l'Amérique du Nord, quoique pouvant compter sur une population "interne" de 270 millions d'habitants, ne se retrouve pas si bien situće sur les routes internationales. Le tableau 1 illustre les effets de ces différences; en effet, malgré une population quatre fois moins nombreuse, on retrouve plus de touristes étrangers en France qu'aux Etats-Unis. Ainsi en France on accueillait en 1987, 37 millions de touristes étrangers contre seulement 29,7 millions aux États-Unis. Cela représente, au prorata de la population, cing fois plus de touristes étrangers qu'aux Etats-Unis. Cette situation s'explique par le fait qu'il n'existe qu'un seul pays émetteur de touristes près des EtatsUnis (le Canada) et que ce pays a une population relativement faible. A l'opposé, la France a une clientèle beaucoup plus diversifiée puisque le principal pays émetteur de touristes (l'Allemagne) ne représente que $24 \%$ de la clientèle étrangère totale.

En ce qui concerne le Canada et le Québec, on constate un apport important de touristes étrangers provenant à $85 \%$ du méme pays, soit les États-Unis. La proximité de l'important marché américain est bien sûr la principale raison de ce phénomène. Le tableau 2 permet de visualiser l'expansion considérable des voyages des touristes français vers l'Amérique du Nord depuis quelques années.

\footnotetext{
* Pierre Bellerose est consultant en tourisme
}

\begin{tabular}{|c|c|c|c|}
\hline \multicolumn{4}{|c|}{$\begin{array}{l}\text { TABLEAU } 1 \\
\text { Nombre de touristes }{ }^{(1)} \text { étrangers au Canada, } \\
\text { aux États-Unis et en France pour l'anné } 1987\end{array}$} \\
\hline Pays & $\begin{array}{l}\text { Nombre total } \\
1987\end{array}$ & $\begin{array}{c}\text { En provenance du principal } \\
\text { pays émetteur }\end{array}$ & $\begin{array}{l}\text { Nombre total de } \\
\text { touristes etrangers } \\
\text { par } 100 \text { habitants }\end{array}$ \\
\hline France $^{(2)}$ & 37,0 millions & 8,8 millions (Allemagne) & 67 \\
\hline Etats-Unis ${ }^{(2)}$ & 29,7 millions & 12,4 millions (Canada) & 12 \\
\hline $\begin{array}{l}\text { Canada } \\
\text { Total }^{(2)}\end{array}$ & 15,0 millions & 12,7 millions (Érats-Unis) & 58 \\
\hline Québec $(1986)^{134}$ & 3.5 millions & 3,0 millions (Etats-Unis) & 54 \\
\hline
\end{tabular}

(1) Exclut les excursionnistes (moins d'une nuitée)

(2) Source: OCDE. Politique du Tourisme et Tourisme International dans les pays membres de I'OCDE. Paris 1988

(3) II s’agit du nombre de visites-provinces pour 1986. Source: Patrick Cluzeau. Le Québec touristique. Indicateurs sur les marchés et sur les secteurs touristiques de 1980 à 1986. Compilation spéciale de l'auteur. On estime généralement à $5 \%$ l'augmentation entre 1986 et 1987: il s'agit d'approximations.

Ainsi, entre 1980 et 1987 on constate une augmentation substantielle pour tous les marchés soit $70 \%$ aux Etats-Unis, $62 \%$ au Canada et $67,7 \%$ au Québec seulement. Ces données illustrent bien le pôle touristique qu'est le Québec pour les touristes français en Amérique du Nord. En effet, les 152000 touristes français qui ont passé en 1987 à tous le moins une partie de leur séjour au Québec, représentent plus de $80 \%$ de tous les touristes français venus au Canada. Ce phénomène est relić bien entendu à la proximité linguistique et culturelle qui existe entre le Québec et la France mais aussi à tous les échanges qui sont survenus depuis 20 ans comme ceux amorcés par exemple par I'OFQJ.

En fait, en considerant qu'un certain nombre de touristes français visitent à la fois les États-Unis et le Québec, on peut affirmer qu'au moins un Français sur quatre en voyage en Amérique du Nord accomplit une partie de son sếjour au Québec. Le tableau 2 nous montre aussi que la croissance observée sur tous les marchés nord-américains s'est faite entre 1985 et 1987 . Les premiers indices connus pour 1988 laissent présager la continuation de cette augmentation pour 1988.

Quant à lui. le tableau 3 illustre l'évolution des flux touristiques de l'Amérique du Nord vers la France. Comme on pouvait s'y attendre, ce sont les Etats-Unis qui apparaissent comme le marché le plus important avec 1,8 million de touristes en France (1987). Loin derrière suivent le Canada ( 32700 ) et le Qué- bec (148 500). Le cas du Québec et du Canada est intéressant à souligner par le fait qu'il est le marché avec la plus forte croissance entre 1980 et 1987 . De plus, le nombre de Québécois vers la France $n^{7} a$ pas connu de fortes fluctuations à la baisse comme ce fut le cas pour les Américains. En effet, ni les fluctuations des taux de change. ni le terrorisme, ni la crise économique de 1982 n'ont empéché de maintenir, année après année, une croissance soutenue (sauf pour une légère baisse depuis 1985).

À l'inverse, les voyages des Américains en France ont connu des variations très brusques durant la période 1980-1987. Ainsi on observe une augmentation impressionnante de $133 \%$ entre 1980 et 1985 pour connaître par la suite une brusque chute de $35 \%$ entre 1985 et 1987. En comparaison, le Québec a connu une hausse de $93,9 \%$ entre 1980 et 1985 et une baisse minime de $6 \%$ entre 1985 et 1987. Cette situation laisse entrevoir un phénomène intéressant. En effet, alors qu'en 1980 le nombre de Québécois représente $40 \%$ du total canadien, cette proportion passe à $46 \%$ en 1987 . Le marché québécois est done celui qui est le plus en expansion en Amérique du Nord et qui est le plus important au prorata de la population pour la France. Finalement, il est intéressant de noter que la baisse des Canadiens ( $13 \%$ en France entre 1985 et 1987) est plus importante que celle des Québécois. Il apparaît à la lecture des données disponibles que depuis 1985 surtout, les Québécois se singularisent par leur fidélité au produit "France". 


\section{Le terrorisme et \\ le taux de change}

Plusieurs facteurs importants sont venus influencer les déplacements transatlantiques dans les années 80 , telles la crise économique majeure de 1982, la déréglementation, la concurrence accrue des transporteurs aériens mais pour les fins de cet article nous nous en tiendrons aux deux phenomenes qui nous apparaissent les plus importants dans ce cas-ci, soit le terrorisme et les fluctuations des taux de change. Les analyses du tableau 3 nous révèlent une baisse de près d'un million de visiteurs nord-américains vers la France entre 1985 et 1987 . Cette chute est reliée en grande partic à ces deux phénomènes.

En effet, même si aucune étude empirique sérieuse n'est venue entériner l'hypothèse de I'impact du terrorisme européen de 1986 sur les performances touristiques de la France, il est indéniable selon les experts que le traitement médiatique aux États-Unis du phénomène "terrorisme" a provoqué un impact négatif impressionnant. En soi, le terrorisme n'a aucun effet sur le tourisme tant qu'il n'est pas repris par la télévision et les journaux. En 1986, les événements survenus à Paris, surtout, ont retenu fortement l'attention des médias américains,... et lorsqu'on connait l'influence de ces médias sur les populations américaines, on comprend plus facilement une partie de la baisse observée entre 1985 ct 1987.

L'autre facteur important qui explique les fluctuations des flux touristiques transatlantiques est le taux de change. Le tableau 4 nous montre les variations apparues en ce qui concerne la valeur du franc français en dol= lar canadien et américain. On remarque qu'entre 1980 et 1985 la valeur du franc français en dollars constants (voir tableau 4b) a chuté de près de $40 \%$. A partir de 1985 , nous remarquons le raffermissement tout aussi spectaculaire pour arriver en 1987 à un niveau semblable à 1980 .

En général, les fluctuations des taux de change ont peu d'impact sur le tourisme si les changements sont modérés et se font sur le long terme. Mais des variations importantes comme celles observées au tableau 4 ont des répercussions très fortes. En faisant le parallèle entre les variations du nombre de touristes nord-américains en France (tableau 3) et les fluctuations du taux de change, on remarque immédiatement la corrélation entre oes deux phénoménes. Parallèlement à cela. le nombre de Français en Amérique du Nord a stagné entre 1980 et 1983 pour augmenter quelque peu en 1984 et fortement à partir de 1986 (tableau 2).

En conclusion, il apparait donc que la croissance observée du nombre de touristes nord américains en France a été quelque peu artìficielle puisqu'aidée par la baisse du franc français. Aussitôt que le franc français s'est raffermi, on a assisté à une décroissance de

TABLEAU 2

Nombre de touristes français venus en Amérique du Nord entre 1980 el 1987

\begin{tabular}{|c|c|c|c|}
\hline \multirow[t]{2}{*}{ Année } & \multicolumn{2}{|c|}{ Au Canada } & \multirow[t]{2}{*}{ Aux États-Unis } \\
\hline & Au Québec ${ }^{(1)}$ & Total & \\
\hline 1980 & 91110 & 115800 & 320000 \\
\hline [98] & 95500 & 118800 & 330000 \\
\hline 1982 & 95300 & 118.100 & 310000 \\
\hline 1983 & 71000 & 90700 & 204812 \\
\hline 1984 & 87300 & 110800 & 334000 \\
\hline 1985 & 84900 & 108400 & 335564 \\
\hline 1986 & 114900 & 140300 & 439611 \\
\hline 1987 & 152000 & 187600 & 544435 \\
\hline $1980-87$ & $+67,6 \%$ & $+62 \%$ & $+70 \%$ \\
\hline
\end{tabular}

Source: Organisation Mondiale du Tourisme et Statistique Canada

(1) Il s'agit de visites-provinces. C'est un indicatcur plus significatif que les simples entrices aux frontieres. Ainsi un Français qui arrive par Toronto mais qui accomplit une partie de son séjour au Québec est inclus dans le total.

TABLEAU 3

Nombre de touristes nord-ambericains venus en France entre 1980 eq 1987

\begin{tabular}{lrrr}
\hline Annce & \multicolumn{2}{c}{ Du Canada } & Des Etats-Unis ${ }^{[3)}$ \\
\cline { 2 - 3 } & Du Québec & \\
\hline 1980 & 81600 & 205000 & 1190000 \\
1981 & 77500 & 196000 & 1372000 \\
1982 & 81000 & 217000 & 1355000 \\
1983 & 87200 & 270000 & 2505000 \\
1984 & 125900 & 325000 & 2539000 \\
1985 & 158200 & 377000 & 2778000 \\
1986 & 154000 & 346000 & 1668000 \\
1987 & 148500 & 327000 & 1802000 \\
\hline $1980-87$ & $+82 \%$ & $+59 \%$ & $+51.4 \%$ \\
\hline
\end{tabular}

(1) Il s'agit de visites-pays. Ce concept a été développé ćtant donné que de nombreux touristes québecois revenant de pays autres que les États-Unis en avaient visité plusieurs durant un même voyage. Source: Statistique Canada.

(2) Source: Statistique Canida

(3) Sounce: Organisation Mondiale du Tourisme.

TABLEAU 4

Valeur d'un franc françuis en dollars canadiens et américains entre 1980 et 1987

A. En dollars courants

\begin{tabular}{lccccccccc}
\hline PAYS & & 1980 & 1981 & 1982 & 1983 & 1984 & 1985 & 1986 & 1987 \\
\hline CAN. & $(\$)$ & 0.265 & 0.206 & 0.183 & 0.149 & 0.138 & 0.185 & 0.214 & 0.235 \\
E.-U. & $(\$)$ & 0.221 & 0.174 & 0.149 & 0.120 & 0.104 & 0.132 & 0.155 & 0.180 \\
\hline
\end{tabular}

B. En dollars constants $(1980=100)$

\begin{tabular}{llllllllll}
\hline PAYS & & 1980 & 1981 & 1982 & 1983 & 1984 & 1985 & 1986 & 1987 \\
\hline CAN. & $(\$)$ & 100.0 & 78.7 & 70.3 & 59.5 & 56.6 & 77.2 & 87.8 & 94.4 \\
E.-U. & $(\$)$ & 100.0 & 80.7 & 72.7 & 62.2 & 55.7 & 72.4 & 85.2 & 98.5 \\
\hline
\end{tabular}

Source: Travel and Tourism Analyst, no. 3, 1988. pp. 90 et 91

ec marché. Bien malin qui saura prévoir le sort du marché français dans l'avenir mais celui qui osera s'attaquer ầ de telles prévi- sions devra en premier lieu évaluer la santé de l'economie française, et donc, par ricochet, celle de sa monnaie. 